\title{
ROBUST BILATERAL TRADE AND MEDIATED BARGAINING
}

\author{
Jernej Čopič \\ UCLA
}

\author{
Clara Ponsatí \\ Institut d'Anàlisi Econòmica, CSIC
}

\begin{abstract}
We consider bilateral trade problems subject to incomplete information on the reservation values of the agents. We address negotiations where the communication of proposals takes place through the filter of a third party, a mediator: traders submit proposals over continuous time to the mediator that receives bids and keeps them secret until they are compatible. A regular robust equilibrium (RRE) is an (undominated) ex post equilibrium where (with sufficient delay) all compatible traders reach agreement. We present a characterization of RRE for risk-neutral traders that discount the future at the same exponential rate. We show how to compute RRE strategy profiles, and we explicitly display the unique one where agreements split the net surplus in equal shares. Our results support the claim that bargaining through a mediator is an effective procedure to promote efficiency. (JEL: C78, D02, D74, D82)
\end{abstract}

\section{Introduction}

Consider the classical bilateral trade problem under two-sided incomplete information. A buyer and a seller wish to exchange an indivisible object, while the cost of the seller and the valuation of the buyer are private information. Suppose that a price is bargained non-cooperatively over time and that agents are impatient. It is well known that full efficiency-immediate agreement whenever the valuation exceeds the cost-is impossible: ${ }^{1}$ regardless of the procedure agreements must be delayed. Unless the distribution of valuations assures that a price acceptable for every cost and valuation exists at the onset, bargainers have a double reason to delay their concessions. First, time is a screening device; with delay agents signal that they need a good deal while they explore how much the opponent can give in. Second, the incentive for delay is reinforced by the fear that an early concession reveals weakness and opens the door to exploitation by the opponent. The compounded result of the two effects are very inefficient outcomes. In this article we

Acknowledgments: Financial support from Ministerio de Educación y Ciencia (SEJ2006-02079) is gratefully acknowledged.

E-mail addresses: Čopič: jcopic@econ.ucla.edu; Ponsatí: clara.ponsati@iae.csic.es

1. Myerson and Satterthwaite (1983) prove this statement for risk neutral environments with independent valuations. 
show that mediation is an effective procedure to decrease this inefficiency because the filtering of information through a mediator cancels the second effect. With face to face bargaining ruled out, agents are protected from exploitation and can therefore concede at the (constrained) optimal speed that allows the realization of all gains from trade.

The mediated bargaining (MB) game is as follows: in continuous time, traders submit their proposals to a third party, the mediator. The key feature of mediation is that the direct information flow between the agents is minimized. ${ }^{2}$ The mediator's role is to receive bids, making them public only when they become compatible. As soon as the seller's price bid is as great as the buyer's, the mediator announces agreement and the game ends with trade at the agreed price. Thus, the traders recognize their net surplus only upon agreement, and at that moment the game is over.

We are interested in equilibrium predictions of the MB game that are robust and deliver a regular pattern of behavior. An equilibrium is robust if it does not rely on the details of the information structure. By regular behavior we mean that traders follow a smooth pattern of mutual concessions which leads to agreement and trade for all compatible pairs-namely, when the valuation of the buyer is greater than the cost of the seller. Thus, our focus of attention are (Pareto undominated) ex post (Nash) equilibrium profiles of the MB game in regular strategies. An ex post equilibrium is robust because strategies must be mutual best responses for all realization of the opponent type. Consequently they are belief independent: neither common priors nor common knowledge of these are necessary to predict behavior, and changes in the details of the information structure are inconsequential. Because agents can reconsider their plans in response to what they learn along play a requirement of sequential rationality seems natural. It is, however, vacuous because the filtration of information through the mediator assures that players never observe off-equilibrium histories.

Čopič and Ponsatí (2008a) presents a detailed exploration of the MB game for rather general environments. There we provide a full characterization of regular ex post equilibrium profiles, and we prove their existence by construction. In what follows we limit attention to risk neutral traders with identical time preferences. In such environment, under complete information, the natural prediction is an agreement allocating equal shares of the surplus. This outcome prevails in the celebrated Rubinstein game (as delay between alternating offers vanishes), ${ }^{3}$ and it is also the common prescription of most bargaining solutions. The main result that we present in the sequel establishes the existence

2. Practitioners emphasize that hearing the parties separately is crucial for effective mediation. See Dunlop (1984).

3. See Binmore, Rubinstein, and Wolinsky (1986). 
and uniqueness of a robust regular equilibrium where all compatible trading pairs reveal their types (after some delay) and reach an agreement that splits the net surplus equally. This equilibrium has a closed form expression of remarkable simplicity, and it is the unique one where strategies are linear in types.

In summary, our results are noteworthy in two respects: First, we confirm the intuition that restricting direct communication between the agents stimulates agents to reveal their willingness to trade and increases efficiency. This supplies sound theoretical ground for the widespread use of mediation in conflict resolution, and also justifies similar procedures that are used in practice in other contexts. For example, in the limit order book of the Paris Bourse, hidden orders are allowed in order to increase the efficiency of the exchange. Second, we supply a tractable model of dynamic bilateral bargaining under two-sided uncertainty in which straightforward criteria select a unique and simple prediction.

The rest of the article is organized as follows. Section 2 discusses related literature. Section 3 describes the bilateral trade problem and the MB game. In Section 4 we characterize robust regular equilibrium (RRE), and we display the unique one which splits the net surplus in equal shares. We conclude and discuss extensions in Section 5.

\section{Relation to the Literature}

The MB game was first explored by Jarque, Ponsatí, and Sákovics (2003). There the set of possible agreements is assumed to be discrete. In this formulation the set of perfect Bayesian equilibria contains a great variety of strategy profiles, none of them ex post.

The present work also relates to the literature on non-cooperative bargaining under incomplete information. ${ }^{4}$ When impatient agents with private reservation values bargain non-cooperatively over time, they must learn what aspirations are reasonable before they are ready for an agreement. In bargaining with twosided uncertainty, learning is a double-edged sword: When an agent learns of her opponent's readiness for agreement, such knowledge may increase the agent's aspirations. This generates incentives to reveal private information very slowly, so that the scope for useful credible communication is severely limited, if not inexistent.

The Coase conjecture is at the root of this problem: Under one-sided uncertainty, if the sequence of proposals can be arbitrarily fast and equilibrium strategies are Markovian, the uninformed party gives up all the surplus immediately. ${ }^{5}$ Under

4. See discussions and references in Ausubel, Cramton, and Denekere (2002).

5. See Gul and Sonnenschein (1988). 
two-sided uncertainty, when the Coase conjecture applies after a fully revealing move, this move is extremely costly. As a consequence, as the time interval between proposals vanishes the probability of agreement vanishes too. Thus, (constrained) efficiency requires either that agents play history dependent strategies, or that their ability to make/receive proposals is limited. Admati and Perry (1987) take the latter approach. They assume that players can commit to not listen to counteroffers for the time elapse of their choice. Then Markovian equilibria exists where the uninformed side obtains a positive share of the surplus. Extending this approach to two-sided uncertainty, Cramton (1992) displays a separating equilibrium where the weaker side reveals her type and the continuation evolves as in Admati and Perry. Trade occurs (with delay) whenever there are gains. Because both agents prefer that the opponent makes the first revealing move, the game is effectively played as a war of attrition. Wang (2000) takes a more drastic short-cut to the war of attrition-he assumes that players cannot make offers until their types have been revealed by initial moves announcing only willingness to talk. The equilibrium outcome coincides with that of our robust regular equilibrium allocating equal net shares. Instead of exploring further constraints on what agents can do, we focus on constraints to what agents observe, while allowing a very flexible extensive form.

\section{Bilateral Trade and Mediated Bargaining}

The Bilateral Trade Problem. A seller $(i=s)$ and a buyer $(i=b)$ bargain over the price $p \in[0,1]$ for an indivisible good. Bargaining takes place over continuous time, $t \in[0, \infty]$, and agents discount the future exponentially. When an agreement to trade at price $p$ is reached on date $t \geq 0$, the seller's payoff upon trading at price $p$ at $t$ is $e^{-t}\left(p-v_{s}\right)$ and the buyer's is $e^{-t}\left(v_{b}-p\right)$. The valuations $v_{s}$ and $v_{b}$ are private information. We write $u_{i}\left(p, v_{i}\right)$ to refer to the instantaneous payoffs from trade at price $p$ for a generic player of type $v_{i}$. It is common knowledge that pairs $v=\left(v_{s}, v_{b}\right)$ are drawn from some continuous joint distribution function $G$ with a positive density $g$ over $[0,1]^{2}$. Common knowledge of $G$ is not necessary for our results.

The Game. The mediated bargaining game (MB game) is a dynamic double auction in continuous-time. The traders send private messages, bidding a price for the good, to the mediator. The mediator receives bids, keeps them secret while they are incompatible, and announces the agreement as soon as it is reached. As time goes by, the seller can continuously decrease her bid at any moment, and the buyer can increase it. Thus the agents can revise their bids until they become mutually compatible. When the mediator announces that agreement has been reached, trade takes place at the agreed price, and the game ends. 
Strategies. A strategy $p_{i}(\cdot, \cdot)$ of player $i$ maps her type $v_{i}$ and each instant $t$ into a price bid,

$$
p_{i}(\cdot, \cdot):[0,1] \times[0, \infty) \rightarrow[0,1], \quad i=s, b .
$$

Thus $p_{i}\left(s_{i}, t\right)$ is the price at which $i$ of type $v_{i}$ is willing to trade at $t$. Strictly speaking, a strategy is a function mapping each type and each history into a proposed price at every moment. However, given her type $v_{i}$, the relevant history at time $t$ is only $t$, as the agent is not able to see the bids of her opponent. ${ }^{6}$ The mediator does not allow the players to renege on their offers, thus the strategy of the seller must be (weakly) decreasing in $t$, and the strategy of the buyer must be (weakly) increasing in $t$. Because monotone functions are differentiable almost everywhere this requirement demands that $\partial p_{s}\left(v_{s}, t\right) / \partial t \leq 0$ and $\partial p_{b}\left(v_{b}, t\right) / \partial t \geq$ 0 for all $\left(v_{b}, v_{s}\right) \in[0,1]^{2}$ and for almost everywhere $t$.

Outcomes. Given a pair of types $v$ and a strategy profile $p$ let $\tau(p, v)$ denote the first time that agents agree on the price, that is

$$
\tau(p, v)=\inf \left\{t \mid p_{s}\left(v_{s}, t\right) \leq p_{b}\left(v_{b}, t\right)\right\} .
$$

If two strategies are such that $\tau(p, v)=\tau<\infty$, then agreement between types $\left(v_{s}, s_{b}\right)$ occurs at $\tau$ at price $\left(p_{b}\left(v_{b}, \tau\right)+p_{s}\left(v_{s}, \tau\right)\right) / 2 .^{7}$ If $\tau(p, v)=\infty$, then the outcome of the game is perpetual disagreement.

Equilibria. A pair of strategies $\left(p_{s}, p_{b}\right)$ is an ex post equilibrium if they are mutual best responses for each pair of types $\left(v_{s}, v_{b}\right)$; that is, $p_{s}\left(v_{s}, t\right)=p_{b}\left(v_{b}, t\right)$ if and only if $t$ maximizes $e^{-\tau}\left(v_{b}-p_{s}\left(v_{s}, \tau\right)\right)$ and $e^{-\tau}\left(p_{b}\left(v_{b}, \tau\right)-v_{s}\right){ }^{8}$ At an ex post equilibrium, when a player knows the type of her opponent, she does not want to reconsider her planned behavior. Observe that equilibrium outcomes are ex post individually rational; this is obvious because agents prefer disagreement to negative payoffs at every moment; hence in equilibrium $p_{s}\left(v_{s}, t\right) \geq v_{s}$ and $p_{b}\left(v_{b}, t\right) \leq v_{b}$ for all $t$ and all $v \in[0,1]^{2}$. On the other hand, the filtration of information through the mediator assures that off-equilibrium histories either end the game or are unobservable to the opponent. Hence own deviation from an ex post equilibrium cannot be optimal at any $t$, and therefore sequential rationality is assured.

6. Because the time is continuous, a detailed specification of admissible strategies requires some care. The interested reader is referred to Čopič and Ponsatí (2008).

7. This sharing rule is inconsequential. In equilibrium, whenever $\tau(p, v)>0$, the agreed price is $\pi(p, v)=p_{s}\left(v_{s}, \tau(p, v)\right)=p_{b}\left(v_{b}, \tau(p, v)\right)$, and the outcome of the game for $v$ and $p$ is $(\pi(p, v), \tau(p, v))$.

8. Alternatively $u_{i}\left(\pi(p, v), v_{i}\right) e^{-\tau(p, v)} \geq u_{i}\left(\pi\left(p_{i}^{\prime}, p_{j}, v\right), v_{i}\right) e^{-\tau\left(p_{i}^{\prime}, p_{j}, v\right)}, \forall p_{i}^{\prime} \neq p_{i}, i=s, b$, $j \neq i$. 
For each ex post equilibrium profile $p$, a profile $p^{\prime}$ constructed by adding a stand still interval $[0, T)$, that is $p_{i}^{\prime}\left(v_{i}, t+T\right)=p_{i}\left(v_{i}, t\right)$, is an ex post equilibrium as well, for any $T<\infty$. As the opponent does not concede any positive amount until $T$, no concession prior to $T$ is useful. Regardless of $T$, such strategy profiles $p^{\prime}$ are weakly dominated. We say that an ex post equilibrium is undominated if it does not have a stand still interval.

Among undominated ex post equilibria, we wish to investigate those where the mediator plays an effective role in promoting agreements: a regular robust equilibrium (RRE) is an undominated ex post equilibrium where all compatible types reach agreement at some finite date. We say that an RRE allocates equal net shares if all pairs of compatible types agree to split their net surplus equally, that is to trade at price $\left(v_{s}+v_{b}\right) / 2$.

Mechanisms and posted prices. A direct revelation mechanism consists of $\pi:[0,1]^{2} \rightarrow[0,1]^{2}$ and $\delta:[0,1]^{2} \rightarrow[0,1]^{2}$, which map reported reservation values into prescribed outcomes as follows. Given reports $\tilde{v}$ the traders are instructed to trade with probability $\delta(\tilde{v})$ at price $\pi(\tilde{v})$; with probability $1-\delta(\tilde{v})$ the mechanism prescribes no trade. A mechanism is ex post individually rational (IR) if trade is prescribed only at acceptable prices, that is, if $\pi(\tilde{v}) \notin\left[\tilde{v}_{s}, \tilde{v}_{b}\right]$ then $\delta(\tilde{v})=0$. It is ex post incentive compatible (IC) if reporting the true valuation is a dominant strategy. A mechanism is said to be IRIC if it is IR and IC. An IRIC mechanism is undominated if there does not exist another IRIC mechanism that Pareto dominates it. A posted price is a mechanism where $\pi(v)=\bar{p}$ for all $v$ and $\delta(v)=1$ if $\bar{p} \in\left[v_{s}, v_{b}\right]$ and $\delta(v)=0$ otherwise. It is obvious that posted prices are IRIC.

For each strategy profile $p$ of the MB game there is a mechanism $\pi^{p}, \delta^{p}$ that delivers equivalent payoffs. It is constructed as follows. Given $p$ and reservation values $v$ agreement is reached at date $\tau(p, v) \in[0, \infty]$; if $\tau(v, p)<\infty$ trade takes place at price $\pi(v, p)$, if $\tau(v)=\infty$ trade does not take place, and payoffs are $\exp \{-\tau(v, p)\}\left(\pi(v, p)-v_{s}\right)$ and $\exp \{-\tau(v, p)\}\left(v_{b}-\pi(v, p)\right)$. Thus the corresponding mechanism is defined by $\pi^{p}(v)=\pi(v, p)$ and $\delta^{p}(v)=\exp \{-\tau(v, p)\}$. We say that $p$ implements $\left(\pi^{p}, \delta^{p}\right)$. An ex post equilibrium implements an IRIC mechanism; incentive compatibility follows by the revelation principle and individual rationality holds because in equilibrium trade takes place at the ex post stage and it is voluntary.

\subsection{Preliminaries}

Conditions for ex post equilibrium (and RRE) can be established based on the properties of the mechanism implemented by equilibria. The following 
proposition due to Hagerty and Rogerson (1987) characterizes IRIC mechanism in terms of distributions over posted prices. ${ }^{9}$

PROPOSITION 1. (IRIC Characterization) A mechanism $(\pi, \delta)$ is IRIC if and only if it is payoff equivalent to a distribution over posted prices.

In addition to IRIC the mechanism implemented by an RRE profile must be undominated. Furthermore the condition that all compatible pairs eventually reach an agreement translates in the additional requirement of full support, that is, if $v_{b}>v_{s}$ then $\delta(v)>0$. The following is immediate.

PROPOSITION 2. An IRIC mechanism $(\pi, \delta)$ is undominated and satisfies full support if and only if there is a positive density $f$ on $[0,1]$, such that $\delta(v)=$ $\int_{v_{s}}^{v_{b}} f(z) d z$ and $\pi(v)=\int_{v_{s}}^{v_{b}} z f(z) d z /\left(F\left(v_{b}\right)-F\left(v_{s}\right)\right)$, where $F$ is the cumulative distribution function implied by $f$.

Our description of RRE crucially relies on this characterization.

\section{Regular Robust Equilibria}

Let us now turn to the necessary and sufficient conditions for RRE strategy profiles. Because RRE are ex post equilibria, agents must bid prices that attain the highest possible payoff, given the type-contingent strategy of the opponent. In other words, given $v_{b}$ the seller of type $v_{s}$ must be choosing optimally the date at which she bids a price equal to $p_{b}\left(v_{b}, t\right)$, and symmetrically for the buyer. Our next result asserts the necessary and sufficient conditions that assure the mutual optimality of these choices.

PROPOSITION 3. (Characterization of RRE) A strategy profile $p$ is a RRE if and only if it is differentiable with respect to $t$ and strictly type monotone and satisfies the following conditions.

1. For all $v$ and for $t$ such that

$$
p_{s}\left(v_{s}, t\right)=p_{b}\left(v_{b}, t\right)
$$

$p_{i}\left(v_{i}, t\right)$ satisfies, for $i=s, b$, the first-order conditions

$$
\begin{gathered}
p_{s}\left(v_{s}, t\right)-v_{s}=\frac{\partial p_{b}\left(v_{b}, t\right)}{\partial t}, \\
v_{b}-p_{b}\left(v_{b}, t\right)=-\frac{\partial p_{s}\left(v_{s}, t\right)}{\partial t} .
\end{gathered}
$$

2. $p_{s}(0,0)=p_{b}(1,0)$.

9. See also Čopič and Ponsatí (2008b). 
Proof. Because an undominated IRIC mechanism with full support is differentiable and strictly type monotone, so must be strategies in an RRE. Consider an RRE profile $p$ and fix the type of player $i$ to be $v_{i}$. Given the strategy of agent $j$ of type $v_{j}, p_{j}\left(v_{j},.\right)$, an equilibrium strategy $p_{i}\left(v_{i},.\right)$ must meet each bid $p_{j}\left(v_{j}, t\right)$ at a date that maximizes $v_{i}$ 's payoff. That is, $p_{i}\left(v_{i}, t\right)=p_{j}\left(v_{j}, \tilde{t}_{i}\left(v_{i}\right)\right)$, where $\tilde{t}_{i}\left(v_{i}\right)=\arg \max _{t \geq 0} e^{-t} u_{i}\left(p, v_{i}\right)$ subject to $p=p_{j}\left(v_{j}, t\right)$. Substituting the constraint, $\tilde{t}_{i}\left(v_{i}\right)=\arg \max _{t \geq 0} e^{-t} u_{i}\left(p_{j}\left(v_{j}, t\right), v_{i}\right)$ at an interior solution $\tilde{t}\left(v_{i}\right)$ must satisfy the first order conditions, namely, equation (2). It is easy to check that for all $v$, payoffs $U_{v}^{s}(t)=e^{-t}\left(p_{b}\left(v_{b}, t\right)-v_{s}\right)$ and $U_{v}^{b}(t)=e^{-t}\left(v_{s}-p_{b}\left(v_{b}, t\right),\right)$ are locally concave at the value of $t$ such that $p_{b}\left(v_{b}, t\right)=p_{s}\left(v_{s}, t\right)$, so that first order conditions are sufficient for optimality.

Condition 2 is necessary to rule out a stand still interval, and observe moreover that a strictly type monotone profile satisfying 1 is undominated.

In the next proposition we show that there is a one-to-one correspondence between RRE and undominated IRIC mechanisms with full support. Hence, Proposition 2 will imply existence and will provide the tool to compute RRE.

PROPOSITION 4. (Implementation and Existence of RRE) Every RRE implements an undominated IRIC mechanism with full support. Conversely, for each undominated IRIC mechanism with full support there is an RRE profile that implements it.

Proof. Fix a RRE profile $p$ and consider the direct revelation mechanism that it induces: That is $\left(\pi^{p}, \delta^{p}\right)$, where $\pi^{p}(v)=\pi(p, v)$ and $\delta^{p}=\exp \{-\tau(p, v)\}$. Because $p$ is an ex post equilibrium, $\left(\pi^{p}, \delta^{p}\right)$ is ex post incentive compatible and individually rational. Because all pairs that produce a positive net surplus reach agreement at a finite date, $\left(\pi^{p}, \delta^{p}\right)$ satisfies full support. Furthermore, because there are no stand-still intervals and by the monotonicity of IRIC mechanisms an RRE implements a mechanism where $\delta^{p}(0,1)=1$. Hence $p$ implements an undominated IRIC mechanism with full support.

The converse is a direct consequence of Proposition 2 and Proposition 3. Take any $(\pi, \delta)$ undominated, IRIC and with full support, and note that it is differentiable and strictly monotone. Let $\tau(v)$ be defined as $\delta(v) \equiv e^{-\tau(v)}$. Observe that $\partial \tau / \partial v_{s}>0, \partial \tau / \partial v_{b}<0$, and $\partial \pi / \partial v_{i}>0$. A RRE that implements a $(\pi, \delta)$ must satisfy

$$
p_{s}\left(v_{s}, \tau(v)\right)=p_{b}\left(v_{b}, \tau(v)\right)=\pi(v) .
$$

For each $v_{i}$ and each $t$ define $\tilde{v}_{j}\left(v_{i}, t\right)$ as the solution to $\tau\left(v_{i}, \tilde{v}_{j}\right)=t$. By the strict monotonicity and differentiability of $\tau, \tilde{v}_{j}\left(v_{i}, t\right)$ is stricty monotone and differentiable function with $\partial \tilde{v}_{j} / \partial t=1 /\left(\partial \tau / \partial v_{j}\right)$. The following strategy 
profile implements $(\pi, \delta)$ :

$$
p_{i}^{\pi, \delta}\left(v_{i}, t\right)=\pi\left(v_{i}, \tilde{v}_{j}\left(v_{i}, t\right)\right), \quad \text { for } i=s, b \text { and } j \neq i .
$$

To check that $p^{\pi, \delta}$ is an RRE simply observe that $\partial p_{i} / \partial t=\partial \pi / \partial v_{j} \cdot \partial \tilde{v}_{j} / \partial t$.

Next we carry out this exercise for the (unique) undominated IRIC mechanism with full support that is linear in types. We emphasize the special relevance of this particular mechanisms, because it is the unique one that prescribes agreements that allocate the net surplus in equal shares, namely, trade at price $\left(v_{b}+v_{b}\right) / 2$, for every pair of compatible traders.

PROPOSITION 5. (The Equal Shares Equilibrium) There is a unique RRE where agreements allocate equal shares of the net surplus. It is given by the following type-contingent strategy profile:

$$
\begin{aligned}
& p_{s}\left(v_{s}, t\right)=\min \left\{1, v_{s}+\frac{e^{-t}}{2}\right\}, \\
& p_{b}\left(v_{b}, t\right)=\max \left\{0, v_{b}-\frac{e^{-t}}{2}\right\} .
\end{aligned}
$$

Proof. Taking a uniform distribution over posted prices in $[0,1]$ yields the mechanism $\pi(v)=\left(v_{b}+v_{b}\right) / 2, \delta(v)=\max \left\{v_{b}-v_{s}, 0\right\}$. Checking that (1) and (2) hold is a straight forward computation. It is also easy to check that no other positive density over $[0,1]$ can sustain $\pi(v)=\left(v_{b}+v_{b}\right) / 2$.

Hence, there is a unique RRE allocating equal shares of the net surplus for every pair of compatible traders. The striking simplicity of this strategy profile (and associated payoffs) should prove quite useful in applications.

\section{Discussion}

It is noteworthy to remark that every (undominated) Bayesian equilibrium in type monotone and regular strategies is an ex post equilibrium. ${ }^{10}$ A Bayesian equilibrium in type monotone and regular strategies must satisfy the following properties: First, all compatible trading pairs reach an agreement at a delayed but finite date. Second, strategies are belief independent so that they are an ex post equilibrium. Therefore they are RRE.

10. See Čopič and Ponsatí (2008a) for a proof. 
Regularity is not an innocuous requirement. Consequently RRE do not exhaust all ex post equilibria. For example, the pair of strategies

$$
\begin{aligned}
& p_{s}\left(v_{s}, t\right)=\left\{\begin{array}{l}
\bar{p}, v_{s} \leq \bar{p}, \\
1, v_{s}>\bar{p}
\end{array}\right. \\
& p_{b}\left(v_{b}, t\right)=\left\{\begin{array}{l}
\bar{p}, v_{b} \geq \bar{p} \\
0, v_{s}<\bar{p}
\end{array}\right.
\end{aligned}
$$

for every $t$, is an ex post equilibrium. With appropriate beliefs at $t>0$, it can also be sustained as a perfect Bayesian equilibrium. In general, ex post equilibria that are not RRE require strategies at which positive masses of types make the same proposal all at the same date, as in the example. These equilibria require a great deal of coordination, which is not robust to trembles. In the example players must exactly coordinate at the arbitrary price $\bar{p}$; if one of the bargainers trembles and proposes $\bar{p}+\varepsilon$ instead of $\bar{p}$, then some types of the opponent would find profitable to deviate upsetting the equilibrium properties of the strategy profile.

The present approach can be applied to address situations with more than two agents. This generalization would be appropriate to address the problems of when to supply, and how to share the cost of a public good when there are many agents. In this case, the mediator can be envisioned as a central agent administering a public account. Individuals pledge their contributions towards the cost of the public good, and can increase their pledge at any time. The mediator assures that contributions are not publicly disclosed until the necessary amount has been pledged. Payments are made only if and when the project is carried out.

Our results may also be interpreted as an analysis of face to face bargaining between agents that do not update their beliefs in response to opponent's offers. This limited revision of beliefs, that might be due to cognitive constraints, delegation, or commitment, means that bargainers learn only what their opponent cannot yield, so their aspirations change smoothly over time.

\section{References}

Admati, Anat R., and Motty Perry (1987). "Strategic Delay in Bargaining." Review of Economic Studies, 54, 345-364.

Ausubel, Lawrence M., Peter Cramton, and Raymond J. Denekere (2002). "Bargaining with Incomplete Information." In Handbook of Game Theory, volume 3, edited by Robert J. Aumann and Sergiu Hart. Elsevier Science.

Binmore, Kenneth, Ariel Rubinstein, and Asher Wolinsky (1986). "The Nash Bargaining Solution in Economic Modelling." Rand Journal of Economics, 17, 176-188.

Cramton, Peter (1992). "Strategic Delay in Bargaining with Two-sided Uncertainty." Review of Economic Studies, 59, 205-225.

Čopič, Jernej, and Clara Ponsatí (2008a). "Optimal Robust Bargaining.” Working paper, http://www.iae.csic.es/ponsati. 
Čopič, Jernej, and Clara Ponsatí (2008b). "Ex post Constrained Efficient Bilateral Trade with Risk Averse Traders," Working paper, http://www.iae.csic.es/ponsati.

Dunlop, John T. (1984). Dispute Resolution. Auburn House.

Gul, Faruk, and Hugo Sonnenschein (1988). "On Delay in Bargaining with One-sided Uncertainty." Econometrica, 56, 155-190.

Hagerty, Kathleen, and William Rogerson (1987). "Robust Trading Mechanisms.” Journal of Economic Theory, 42, 94-107.

Jarque, Xavier, Clara Ponsatí, and József Sákovics (2003). "Mediation: Incomplete Information Barganing with Filtered Communication.” Journal of Mathematical Economics, 39, 803 830.

Myerson, Roger, and Mark Satterthwaite (1983). "Efficient Mechanism for Bilateral Trading." Journal of Economic Theory, 29, 265-281.

Wang, Ruqu (2000). "Separating Equilibria in a Continuous-time Bargaining Model with Twosided Uncertainty." International Journal of Game Theory, 29, 229-240. 